\title{
$S$. Typhimurium virulence changes caused by exposure to different non-thermal preservation treatments using C. elegans
}

\author{
María Sanz-Puiga, Elena Lázaro ${ }^{\mathrm{b}}$, Carmen Armero $^{\mathrm{b}}$, Danilo Alvares ${ }^{\mathrm{b}}$, \\ Antonio Martínez ${ }^{\mathrm{a}}$, Dolores Rodrigo. ${ }^{\mathrm{a}, *}$ \\ ${ }^{a}$ Instituto de Agroquímica y Tecnología de Alimentos (IATA-CSIC), \\ Carrer del Catedràtic Agustín Escardino Benlloch, 7, 46980, Paterna, València, Spain \\ ${ }^{b}$ Department of Statistics and Operations Research, Universitat de València, \\ Carrer Doctor Moliner, 50, 46100, Burjassot, Spain
}

\begin{abstract}
The aims of this research study were: (i) to postulate Caenorhabditis elegans ( $C$. elegans) as a useful organism to describe infection by Salmonella enterica serovar Typhimurium ( $S$. Typhimurium), and (ii) to evaluate changes in virulence of $S$. Typhimurium when subjected repetitively to different antimicrobial treatments. Specifically, cauliflower by-product infusion, High Hydrostatic Pressure (HHP), and Pulsed Electric Fields (PEF).

This study was carried out by feeding $C$. elegans with different microbial populations: E. coli OP50 (optimal conditions), untreated S. Typhimurium, S. Typhimurium treated once and three times with cauliflower by-product infusion, $S$. Typhimurium treated once and four times with HHP and $S$. Typhimurium treated once and four times with PEF.

Bayesian survival analysis was applied to estimate C. elegans lifespan when fed with the different microbial populations considered. Results showed that C. elegans is a useful organism to describe infection by $S$. Typhimurium because its lifespan was reduced when it was infected. In addition, the application of antimicrobial treatments repetitively generated different responses: when cauliflower by-product infusion and PEF treatment were applied repetitively the virulence
\end{abstract}

\footnotetext{
* Corresponding author

Email address: lolesra@iata.csic.es (Dolores Rodrigo.)
} 
of $S$. Typhimurium was lower than when the treatment was applied once. In contrast, when HHP treatment was applied repetitively, the virulence of $S$. Typhimurium was higher than when it was applied once. Nevertheless, in all the populations analyzed treated $S$. Typhimurium had lower virulence than untreated $S$. Typhimurium.

Keywords: Antimicrobial; Bayesian survival analysis; High Hydrostatic Pressure; Pulsed Electric Fields.

\section{Introduction}

Salmonella spp. are among of the most important foodborne pathogens, causing about 155000 salmonellosis-related deaths each year worldwide (Majowicz et al. 2010). Salmonellosis is a zoonotic infection transmitted to humans

5 through the fecal-oral route, the primary source of transmission being infected eggs and egg-derived products, meat, poultry, milk and milk-derived products, fruits and vegetables (Gómez-Aldapa et al., 2012). Salmonella enterica serovar Typhimurium ( $S$. Typhimurium) and $S$. Enteritidis are currently the most usual serotypes related to salmonellosis outbreaks $(20.2$ and $39.5 \%$ of the reported cases, respectively) (EFSA, 2015).

Different kinds of preservation treatments have been developed to reduce or eliminate these pathogens in food products, inclunding the addition of bioactive substances from nature or from agroindustrial by-products with antimicrobial effect (O'Shea et al., 2012; Viuda-Martos et al., 2008) or the application of

15 non-thermal treatments such as High Hydrostatic Pressure (HHP) (BarbosaCánovas et al., 2008; Rendueles et al., 2011) and Pulsed Electric Fields (PEF) (Mosqueda-Melgar et al. 2012, Saldaña et al., 2014). However, such treatments can have important drawbacks because their repeated use could generate serious antimicrobial resistance problems (Kisluk et al., 2013, Vanlint, 2013).

Nevertheless, the higher consumption of fresh fruits and vegetables recorded in recent years has been associated with an increase in foodborne disease out- 
breaks (Olaimat and Holley, 2012). This is a very important issue that requires much more research work on infection by foodborne pathogens and procedures to combat them, particularly Salmonella spp.

Caenorhabditis elegans (C. elegans) is a very useful experimental organism. It belongs to a nematode species that inhabits soils around the world (Hope, 1999). Its maintenance in the laboratory is easy and cheap because it has a short lifespan, approximately 21 days, with a hermaphroditic life cycle of around three days and a high quantity of offspring (more than 300). In addition, it has a transparent body, which allows observation of all of its organs, and it is fed with Escherichia coli cells as they are eaten by the worms in optimal laboratory conditions (Leung et al., 2008). Moreover, the main physiological processes and stressed responses of higher organisms such as humans are retained in C. elegans. For all of these reasons, C. elegans is a useful organism for analyzing the effects of foodborne pathogens in host organisms and studying their virulence factors.

The aim of this paper is twofold: (i) to describe the effect of $S$. Typhimurium on $C$. elegans organisms, and (ii) to evaluate the development of microbial resistance of $S$. Typhimurium to various antimicrobial treatments applied consecutively, as well as to assess the virulence of the changes observed. For both objectives, the lifespan of the nematodes was analyzed statistically by means of Survival Analysis, specifically Cox Proportional Hazards models (Cox and Oakes, 1984, Cox, 1992, Ibrahim et al., 2001). The first study was performed to assess the effect of $S$. Typhimurium on the host organism. The remaining three studies were conducted to quantify microbial resistance and virulence changes as a consequence of three different preservation treatments: cauliflower by-product infusion, HHP and PEF. Bayesian inference was used to estimate all models thus providing a suitable framework to deal with problems with multiple comparisons and many uncertainties. WinBUGS software (Lunn et al. 2000) was used to simulate the Markov chain whose stationary distribution is 50 the relevant posterior distribution of the parameters of interest from which all relevant inferences are derived. 


\section{Material and methods}

\subsection{Bacterial strain}

A freeze-dried pure culture of $S$. Typhimurium was provided by the Spanish

55 Type Culture Collection (CECT 443). It was rehydrated with tryptic soy broth (TSB) (Scharlab S. A., Barcelona, Spain) and incubated at $37^{\circ} \mathrm{C}$ with continuous shaking (Model 6001173, Selecta Unitronic, Barcelona, Spain) for $14 \mathrm{~h}$. Cells were later centrifuged (Beckman Avanti J-25, USA) twice at $2450 \mathrm{~g}$ and $4^{\circ} \mathrm{C}$ for $15 \mathrm{~min}$ and re-suspended in TSB. Finally, cells were transferred in TSB

${ }_{60}$ with $20 \%$ of glycerol and dispensed to a final concentration of $10^{8} \mathrm{cfu} / \mathrm{mL}$ in 2 $\mathrm{mL}$ vials, which were frozen and stored at $-80^{\circ} \mathrm{C}$.

\subsection{Cauliflower by-product infusion as a natural antimicrobial}

Dehydrated cauliflower by-product (from leaves of cauliflower plants) was provided from primary production of TRASA S.L. It was washed in the laboratory to eliminate contaminants, and then dried, crushed, and homogenized (Brandi et al. 2006). Cauliflower by-product 5\% (w/v) infusion was obtained by boiling it in buffered peptone water $(0.1 \%(\mathrm{w} / \mathrm{v}))$ for $30 \mathrm{~min}$. The infusion was later centrifuged at $2450 \mathrm{~g}$ for $15 \mathrm{~min}$ at $4^{\circ} \mathrm{C}$ and filtered three times (Whatman filter $11 \mu \mathrm{m}$ pore size, Whatman filter $2.5 \mu \mathrm{m}$ and $0.45 \mu \mathrm{m}$ PVDF syringe filter

70 to sterilize, consecutively). $30 \mathrm{~mL}$ of cauliflower by-product infusion $5 \%$ (w/v) was inoculated with $1 \mathrm{~mL} S$. Typhimurium overnight culture $\left(10^{8} \mathrm{cfu} / \mathrm{mL}\right)$ and was incubated at $37^{\circ} \mathrm{C}$ for five hours. The procedure was performed three times. The $S$. Typhimurium population was recovered between each antimicrobial treatment by growing in TSB overnight with continuous shaking. The $S$.

75 Typhimurium concentration was evaluated before and after each antimicrobial treatment by serial dilution in buffered peptone water $(0.1 \%(\mathrm{w} / \mathrm{v}))$ and plate count in TSA (Scharlau, Scharlab). The plates were incubated at $37^{\circ} \mathrm{C}$ for 24 hours. The number of surviving microorganisms obtained after each treatment permits to evaluate the microbial resistance development. Differences in resis-

so tance to infusion were observed between subpopulation exposed once and three times to the antimicrobial (one log reduction and one log growth, respectively). 


\subsection{HHP treatment}

$S$. Typhimurium with an initial concentration of $10^{8} \mathrm{cfu} / \mathrm{mL}$ was treated with HHP (250 MPa five minutes). This is a sublethal treatment to $S$. Typhimurium that causes, mainly, cellular damage (Sanz-Puig et al. 2017). Between HHP treatments, the bacteria were grown in TSB and continuously shaken at $37^{\circ} \mathrm{C}$ overnight to recover the damaged cells. The procedure was performed four times. Before and after HHP treatments, the microbial population was evaluated by serial dilution in buffered peptone water $(0.1 \%(\mathrm{w} / \mathrm{v}))$ and plate count in TSA. The TSA plates were incubated at $37^{\circ} \mathrm{C}$ for 24 hours. The resistance was determined in terms of number of survival microorganisms after each HHP consecutive treatment. As in the case of cauliflower infusion, differences in resistance were observed between subpopulations (2.5 and 0.7 log cycles of microbial reduction for $S$. Typhimurium exposed once and four times to HHP treatment). HHP treatments were applied using EPSI NV equipment (Temse, Belgium) (Pérez et al., 2007a).

\subsection{PEF treatment}

The initial $S$. Typhimurium population $\left(10^{8} \mathrm{cfu} / \mathrm{mL}\right)$ was subjected to PEF treatment $(30 \mathrm{kV} / \mathrm{cm}-300 \mu \mathrm{s})$. Between PEF treatments, the bacteria were grown in TSB overnight with continuous shaking at $37^{\circ} \mathrm{C}$. The procedure was performed four times. Before and after each PEF treatment, the $S$. Typhimurium concentration was evaluated by plate count in TSA and serial dilution in buffered peptone water $0.1 \%(\mathrm{w} / \mathrm{v})$ and the plates were incubated at $37^{\circ} \mathrm{C}$ for 24 hours. The resistance was evaluated by the number of survival microorganisms after each consecutive PEF treatment. The first and the fourth treatment caused, respectively, 2.9 and $0.70 \log$ cycles of microbial reduction. An OSU-4D batchscale continuous PEF system, designed at Ohio State University, was used to treat the samples (Pérez et al., 2007b).

\subsection{C. elegans studies}

110

C. elegans strain N2 was provided by the College of Biological Sciences, Minnesota University, USA. It was maintained in optimal conditions: plates with 
Nematode Growth Medium (NGM) agar and a bacterial lawn of E. coli OP50 (Stiernagle, 2006). The microbial concentration in the lawn of NGM agar was checked in each treatment, measuring its absorbance and it was similar in all plates. C. elegans was used to evaluate the effect of $S$. Typhimurium on host organisms as well as possible changes of virulence in $S$. Typhimurium populations as a result of repetitive exposure to various antimicrobial treatments: cauliflower by-product infusion, HHP and PEF. For this purpose 250 synchronized young adult nematodes, distributed in 25 plates of 10 worms each, were transferred to NGM agar with a lawn of each of the $S$. Typhimurium populations under study: $S$. Typhimurium, $S$. Typhimurium treated once and three times with cauliflower by-product infusion, $S$. Typhimurium treated once and four times with HHP, and $S$. Typhimurium treated once and four times with PEF. The worms were maintained at $20^{\circ} \mathrm{C}$ throughout all their lifespan (approximately three weeks), examined with a binocular microscope (COMECTA S.A.) at 48 hour intervals, and then transferred to new plates. They were considered dead when they did not move or respond to stimulation. All the experiments included a negative control with $C$. elegans in NGM plates with an E. coli OP50 lawn.

\subsection{Modeling strategy}

The lifespan of $C$. elegans was analyzed with regard to the different $S$. Typhimurium populations considered. Lifespan was defined for each C. elegans individual as the time (in days) from the start of the experiment until it was dead. The design of the study focused on time-to-event data which are typically analyzed in the framework of Survival Analysis (Klein and Moeschberger

135 2003). Statistical survival modeling is mainly characterized by censoring issues. In our study, the survival experiment accounted for interval and right-censored data. Most of the data were interval-censored: the lifespan of each individual was not known exactly, and the only relevant information about it was that its death occurred between two consecutive monitoring times. Nevertheless, rightcensored data was used when the individual died accidentally during the process of changing it from one plate to another one. 
The basic probabilistic elements to describe the stochastic behavior of timeto-event data are the survival function and the hazard function. The survival function, $S(t)$, is the probability that an individual survives longer than a given instant in time, $S(t)=P(T>t), t>0$. The hazard function, $h(t)$, is a temporal rate, not a probability (Andersen et al. 2012 ) (, but it can be interpreted approximately as the chance that a living individual dies instantaneously.

The standard Cox proportional hazards (CPH) model (Cox and Oakes, 1984 Cox, 1992) was used to analyze the lifespan of $C$. elegans for each of the populations considered. This model is expressed in terms of the hazard function of the time-to-event of interest which factorizes into the product of a baseline hazard function, $h_{0}(t)$, and an exponential term that assesses the relative rate associated with a set of covariates $\boldsymbol{x}$ (expressed as the linear combination $\boldsymbol{x}^{\prime} \boldsymbol{\beta}$ ) with regression coefficients $\boldsymbol{\beta}$,

$$
h(t)=h_{0}(t) \exp \left\{\boldsymbol{x}^{\prime} \beta\right\}
$$

We carried out four different CPH analyses according to the type of food with which $C$. elegans was fed. Model 1 is the simplest and describes the effect of $S$. Typhimurium on the $C$. elegans organism. Models 2, 3 and 4 analyze the lifespan of $C$. elegans with regard to the level of virulence of $S$. Typhimurium resulting from the application, in different doses, of antimicrobial treatments based on cauliflower by-product infusion, HHP and PEF, respectively. All the survival models were constructed with Weibull baseline hazard functions and indicator covariates for the type of food population considered.

The statistical analysis of the CPH models was approached from Bayesian inference, which provides a powerful and flexible methodology to quantify all the uncertainties of interest in probabilistic terms and to combine all sources of information (Ibrahim et al. 2001). We assumed prior independence and elicited wide proper prior distributions in order to give all inferential prominence to the data.

The posterior distribution of the parameters of each model was approximated by means of Markov chain Monte Carlo methods (MCMC) using WinBUGS 
software (Lunn et al. 2000). The settings were three Markov chains of 33000

values each after discarding the first 3000 values in order to reach convergence to the posterior distribution. The chains were thinned by storing every three iterations thereby reducing the autocorrelation in the sample. Trace plots of the simulated values of the chains appeared to overlap one another indicating stabilization. Convergence of the chains to the posterior distribution was assessed through the potential scale reduction factor and the effective number of independent simulation draws (Gelman and Rubin, 1992, Kass et al., 1998).

\section{Results and discussion}

The content of this section has been arranged in four parts according to the four studies carried out that compare the lifespan of $C$. elegans between treated and untreated $S$. Typhimurium (control group). All results are presented in terms of posterior quantities concerning median lifespan (time from which $50 \%$ of individuals are still living and the other half have died) and survival probabilities in order to improve their interpretability.

\subsection{S. Typhimurium}

The effect of $S$. Typhimurium infection on $C$. elegans was evaluated via the posterior distribution of the median of the lifespan of the worms according to the type of feed, $S$. Typhimurium and their optimal food E. coli OP50, as well as their subsequent posterior survival probabilities.

Figure 1 1 a shows mean and 95\% credible interval for the posterior distribution of $C$. elegans median lifespan for both populations. C. elegans posterior mean lifespan in optimal conditions is around 6.39 days. This was reduced to about 4.82 days when the nematodes were fed with $S$. Typhimurium. This value is very close to five days, which is the time required by $S$. Typhimurium to achieve complete infection of the intestinal lumen of C. elegans, as described in other research studies (Labrousse et al., 2000, Aballay et al. 2000). The uncertainty of the estimates is assessed through credible intervals for the relevant parameters; 


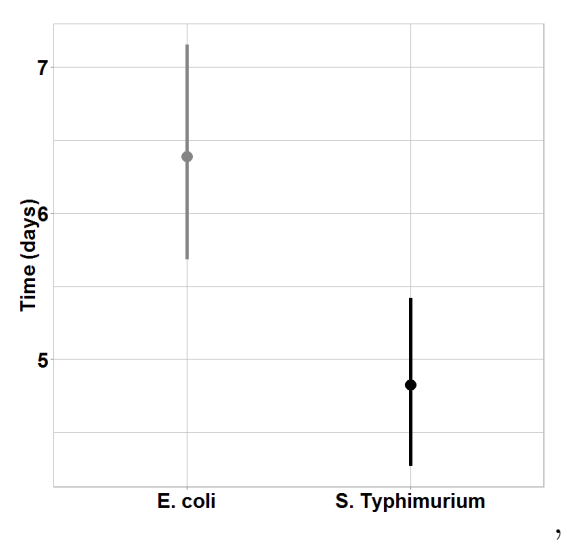

(a)

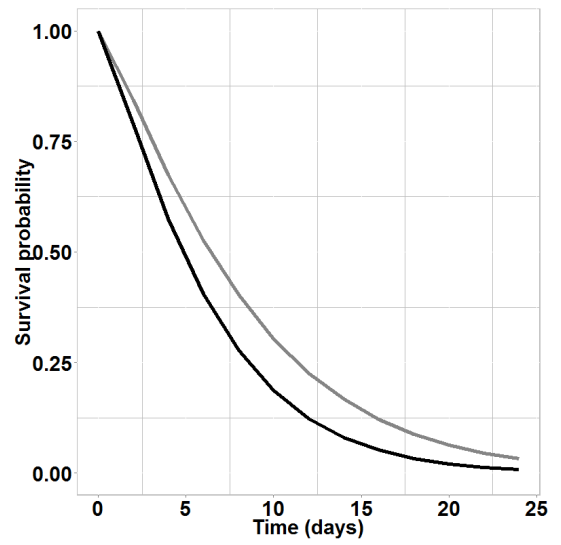

(b)

Figure 1: (a): Posterior expected median lifespan and 95\% CI of C. elegans fed with E. coli OP50 (grey) and with $S$. Typhimurium (black); and (b): posterior expected survival probabilities of $C$. elegans fed with E. coli OP50 (grey) and S. Typhimurium (black).

the $95 \%$ credible intervals $(95 \%$ CI) for the effect of each food indicate that the two distributions cover completely different range of values: [5.68, 7.15] days for $E$. coli and $[4.27,5.42]$ for $S$. Typhimurium. This provides strong evidence that worms fed with $S$. Typhimurium have a shorter lifespan than those fed in optimal conditions (E. coli OP50), such as it has been shown in other studies as (Labrousse et al. 2000). A result, C. elegans seems to be able to highlight the virulence effect of $S$. Typhimurium.

Figure 1 $1 \mathrm{~b}$ presents the posterior mean of the survival probabilities for $C$. elegans fed with each of the microbial populations considered. It is easy to see they show clear differences between the two survival trajectories: probabilities associated with $C$. elegans fed with $S$. Typhimurium are always lower than the corresponding ones for $C$. elegans fed with E. coli OP50. In particular, survival probabilities estimated at days 2,12 , and 22 from the star of the experiment are $0.84,0.22$, and 0.05 for $E$. coli, and $0.78,0.12$ and 0.01 for $S$. Typhimurium, respectively. These results support the idea that survival probabilities of $C$. elegans are always lower in all their life stages when it is infected with $S$. Ty- 
phimurium.

\subsection{S. Typhimurium with cauliflower by-product infusion}

210 from repetitive exposure to cauliflower by-product infusion. Cauliflower byproduct infusion contained bioactive compounds, as a ascorbic acid, carotenoids, flavonoids and, mainly, polyphenols such as galic acid, quercetin or rutin SanzPuig et al., 2015; Vieira, 2013). To accomplish this goal, C. elegans were fed with $S$. Typhimurium exposed once and three times to cauliflower by-product infusion during their entire lifespan, and subsequently compared with C. elegans fed with untreated $S$. Typhimurium (control group).

Figure 2 a shows the posterior expected C. elegans median lifespan and $95 \%$ CI for each of the two cauliflower by-product infusion treatments ( Inf 1 and Inf3 for one and three doses, respectively) as well as for the control group (St). The estimated median lifespan values for $C$. elegans with treatments Inf 1 and Inf 3 are similar, 7.14 and 6.85 days respectively. This is not the case for treatment St, with an estimated value of 4.96 days, which is, as expected, very similar to the one obtained in the previous model. Not only are the punctual estimates

for treated and untreated $S$. Typhimurium very different but also so are their

The next step was to evaluate virulence shifts in $S$. Typhimurium resulting respective $95 \%$ CI values. Specifically, neither of the $\operatorname{Inf} 1([6.37,7.90])$ and Inf3 $([6.20,7.70])$ credible intervals overlaps with the one corresponding to St ([4.43, 5.48]. Hence, when $S$. Typhimurium is exposed to cauliflower by-product infusion repetitively the lifespan increases, so $S$. Typhimurium becomes less virulent than usual, with lower infection effects. This is justified because the posterior probability that the Inf 1 median lifespan is higher than the one for Inf3 is 0.631 , a value that supports a conclusion of identical virulence.

Figure $2 \mathrm{~b}$ presents the estimated survival curves for $C$. elegans fed with microbial populations $\operatorname{Inf} 1$, Inf3, and St. It is hard to distinguish between survival prospects related the two cauliflower treatments. In fact, their respective estimated survival probabilities at 2,12 and 22 days are $0.87,0.26,0.06$ (Inf 1$)$, and $0.86,0.25,0.05$ (Inf3). With regard to the control group, the sur- 


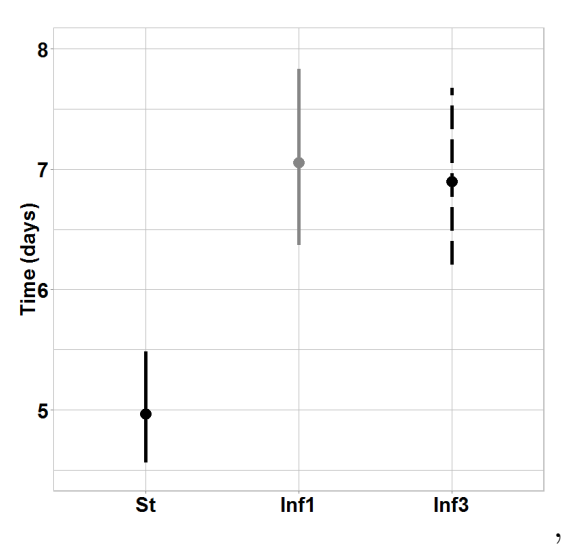

(a)

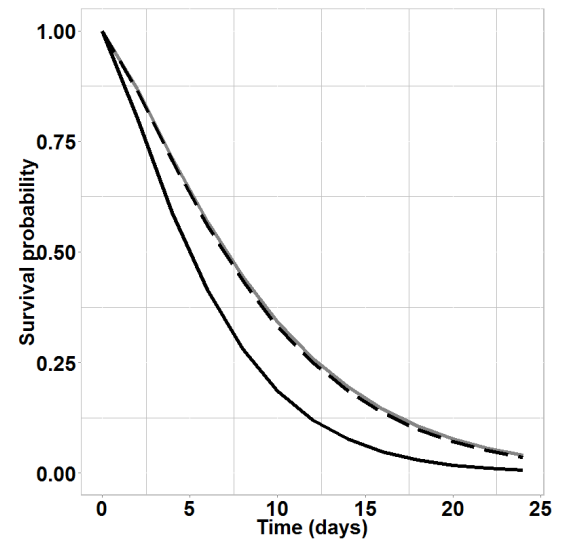

(b)

Figure 2: (a): Posterior expected median lifespan and 95\% CI of C. elegans fed with untreated $S$. Typhimurium (in black and represented as St on the horizontal axis), $S$. Typhimurium treated once with cauliflower by-product infusion (Inf1 in gray) and $S$. Typhimurium treated three times with cauliflower by-product infusion (Inf3 in black dashed); and (b): posterior expected survival probabilities of C. elegans fed with St (black), Inf1 (grey), and Inf3 (black dashed). 
vival trend is lower in relation to both cauliflower treatments, with estimated survival probabilities of $0.80,0.12$, and 0.01 at days 2,12 and 22 , respectively. Therefore, when $S$. Typhimurium is treated with cauliflower by-product infusion its virulence decreases and its infection causes lower effects on $C$. elegans than the ones for control $S$. Typhimurium, thus increasing its survival probabilities throughout its lifespan. However, there are not great differences between Inf 1 and Inf3. It may therefore be concluded that the application of this antimicrobial treatment repetitively does not seem to have consequences that affect the virulence of $S$. Typhimurium .

\subsection{S. Typhimurium with HHP}

Virulence changes in the $S$. Typhimurium microbial population after the application of HHP preservation treatment are now discussed. C. elegans were fed with $S$. Typhimurium treated once and four times with HHP and with untreated $S$. Typhimurium, which was also considered as the control group in this case.

Figure 3 displays the posterior expectation and $95 \%$ CI of the posterior distribution of $C$. elegans median lifespan for both HHP treatments (HHP1 and HHP4 for frequencies one and four, respectively), and the control group (St). $C$. elegans has an estimated median lifespan of 6.44 and 5.81 days in the case of Hhp1 and HHP4, respectively, and 5.15 days for St. The $95 \%$ CI related to St does not overlap the corresponding one for Hhp1 ( $[4.61,5.68]$ and $[5.81,7.07]$, respectively). This is not the case for the comparison between St and HHP4 where a clear overlap can be seen $([5.24,6.38]$ is the $95 \%$ CI for HHP4). Nevertheless, there is a high probability, 0.97, associated with a higher effect of HHP4 with regard to St. In the case of the two HHP treatments the overlap is obvious. Direct comparison between them indicates that Hhp4 increases the virulence of bacteria with regard to HHP1 with a probability of 0.92 .

We can conclude that the application of HHP antimicrobial treatment against $S$. Typhimurium made it less virulent against $C$. elegans than in the case of the control group. However, when HHP treatment was applied repetitively (four 


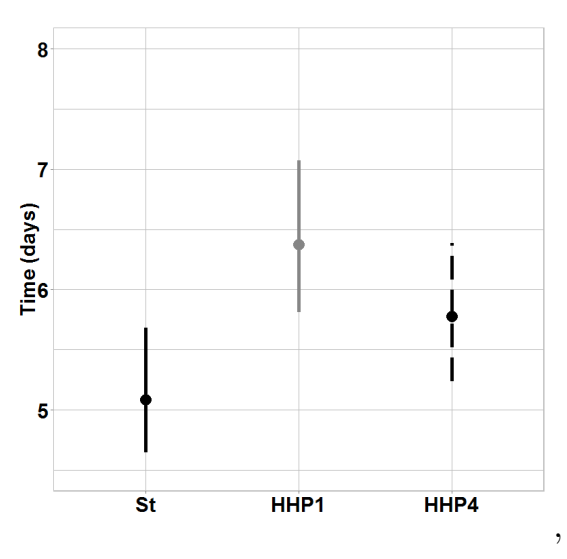

(a)

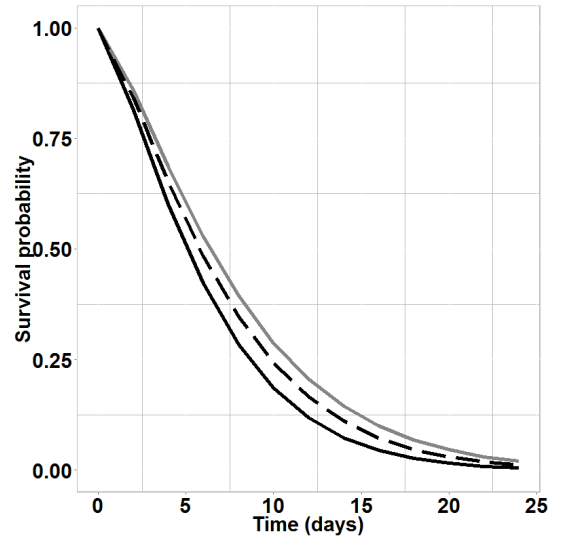

(b)

Figure 3: (a): Posterior expected median lifespan and 95\% CI of C. elegans fed with untreated $S$. Typhimurium (in black and represented as St on the horizontal axis), $S$. Typhimurium treated once with HHP (HHP1 in grey) and $S$. Typhimurium treated four times with HHP (HHP4 in black dashed) ; and (b): posterior expected survival probabilities of C. elegans fed with St (black), HHP1 (grey), and HHP4 (black dashed).

times) the virulence of $S$. Typhimurium increased in comparison with $S$. Typhimurium treated once, although it did not achieve the virulence of untreated $S$. Typhimurium. Although, as far as we concern, there are very few scientific studies about it, the microbial changes produced for recover the cell damage after each treatment could be the responsible of the development of a major microbial virulence. In fact, studies like Van Boeijen et al. (2013) carried out with Listeria monocytogenes high pressure-resistant variants concluded that some variants showed a reduction in microbial virulence and others retained full virulence in a mouse model. The full virulence phenotype showed in the mouse model could be due to the reversion of part of the microbial population to normally cells. These results could open the door to new research studies about the effect of successive applications of HHP treatment on the virulence of $S$. Typhimurium and the subsequent comparison with results for untreated $S$. Typhimurium.

Estimated survival curves for St, HHP1 and HHP4 were based on the poste- 
rior median of C. elegans lifespan (see Figure 3b). The survival prospects are in line with the result discussed in the previous paragraph. The lowest survival probabilities are related to the control group (St), with estimated survival probabilities of $0.81,0.12$, and 0.01 at days 2,12 , and 22 , respectively. The middle survival trend is connected with the HHP4 group, with estimated survival probabilities of $0.86,0.21$, and 0.03 at days 2,12 and 22 , respectively, and the highest one with group HHP1, with probabilities of $0.84,0.17$ and 0.02 estimated at the same days as before. All the survival curves look very similar.

\subsection{S. Typhimurium with PEF}

Finally, the effect of the application of PEF application on the virulence of $S$. Typhimurium against $C$. elegans was considered. C. elegans was fed with $S$. Typhimurium treated once and four times with PEF and with untreated $S$. Typhimurium (control group).

There are considerable differences between the estimated median C. elegans lifespan values for each of the two PEF treatments (PEF1 and PEF4 for frequencies one and four, respectively) (see Figure 4a). The expected median lifespan related to PEF1 is 5.14 days, quite similar to the one for the St group, 4.82 days. The $95 \%$ CI values for PEF1 and St are $[4.55,5.72]$ and $[4.27,5.36]$ days, respectively and the posterior probability that the effect of PEF1 is higher than the one for St is 0.20 , not a value that would justify assuming differences between them. Consequently, the $S$. Typhimurium survivor population after one application of PEF shows similar virulence to the untreated population. With regard to the PEF4 median lifespan, it is noteworthy that the $S$. Typhimurium survivor population after four applications of pulsed electric fields shows the lowest virulence: the $95 \% \mathrm{CI},[5.92,7.38]$ days, has the highest values and does not overlap with the $95 \%$ CI for either St or PEF1. The application of PEF treatment once seems to have no great effect on the microbial virulence of $S$. Typhimurium against $C$. elegans. In contrast, the repetitive application of PEF treatment makes $S$. Typhimurium less virulent against $C$. elegans.

Posterior survival probabilities are displayed in Figure 4p. Survival probabil- 


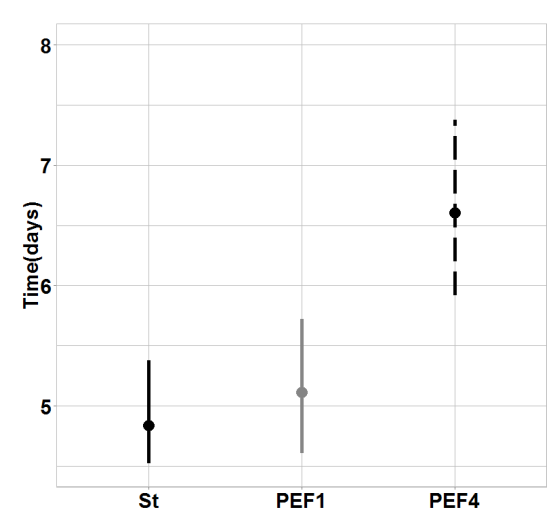

(a)

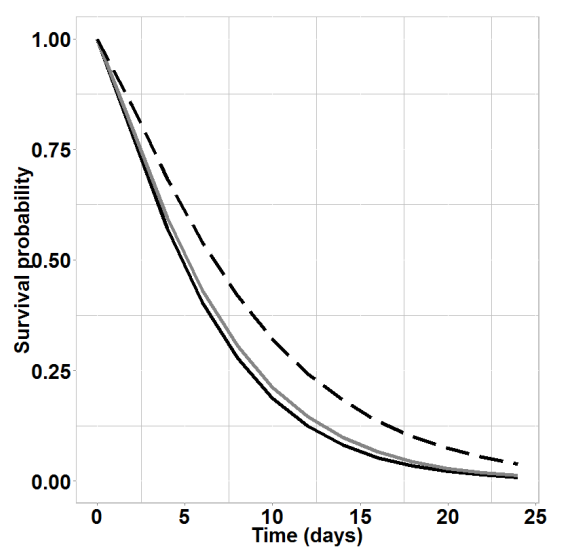

(b)

Figure 4: (a): Posterior expected median lifespan and 95\% CI of C. elegans fed with untreated $S$. Typhimurium (in black and represented as St on the horizontal axis), S. Typhimurium treated once with PEF (PEF1 in grey), and $S$. Typhimurium treated four times with PEF (PEF4 in black dashed); and (b): posterior expected survival probabilities of $C$. elegans fed with St (black), PEF1 (grey), and PEF4 (black dashed).

ities corresponding to St and PEF1 are very similar with estimated probabilities of $0.78,0.12,0.01$ and $0.80,0.15,0.02$ at days 2,12 and 22 , respectively. Survival probabilities related to PEF4 show a different trend with respect to the other two treatments. PEF4 survival probabilities are a little higher throughout the lifespan of C. elegans, with particular probability values of $0.85,0.24,0.05$ at day 2, 12 and 22, respectively.

\section{Conclusions}

The results of this research show that $C$. elegans can be postulated as a useful organism to describe the reduction in lifespan caused by infection by $S$. Typhimurium. In addition, the application of different antimicrobial treatments repetitively had different effects on the virulence of $S$. Typhimurium. In all cases, treated $S$. Typhimurium was less virulent than untreated $S$. Typhimurium. Nevertheless, when $S$. Typhimurium was treated repetitively with 
cauliflower by-product infusion the virulence was kept low after repetitive treatment and when it was treated by PEF, its virulence was lower than when it was treated once. However, S. Typhimurium treated repetitively with HHP had higher virulence than $S$. Typhimurium treated once with HHP.

\section{Acknowledgements}

Sanz-Puig is grateful to the CSIC for providing a contract as a researcher working actively on project AGL 2013-48993-C2-2-R. Lázaro was the recipient of a predoctoral FPU fellowship (FPU/02042/2013) from the Spanish Ministry of Education, Culture and Sports. Alvares' work was supported by Coordination for the Improvement of Higher Level Personnel (BEX: 0047/139), Brazil. The present research work was funded in part by grant AGL 2013-48993-C2-2-R and grant MTM2016-77501-P from the Spanish Ministry of Economy and Competitiveness co-financed with FEDER funds. We are also grateful to TRASA, S.L. for providing the by-product that we worked with.

\section{References}

Aballay, A., Yorgey, P., Ausubel, F. M., 2000. Salmonella typhimurium proliferates and establishes a persistent infection in the intestine of Caenorhabditis elegans. Current Biology. 10(23), 1539-1542.

Andersen, K., Geskus, R. B., de Witte, T., Putter, H., 2012. Competing risks in epidemiology: possibilities and pitfalls. International Journal of Epidemiology. 41 , 861-870. doi:10.1093/ije/dyr213

Barbosa-Cánovas, G. V., Juliano, P., 2008. Food sterilization by combining high pressure and thermal energy. In: Food engineering: Integrated approaches. Springer, pp.9-46. doi:10.1007/978-0-387-75430-7_2.

350 Brandi, G., Amagliani, G., Schiavano, G. F., De Santi, M., Sisti, M., 2006. Activity of Brassica oleracea leaf juice on foodborne pathogenic bacteria. Journal of Food Protection. 69 , 2274-2279. 
Cox, D. R., 1992. Regression models and life-tables. In: S. Kotz, \& N. L. Johnson 355 (Eds.), Breakthroughs in Statistics: Methodology and Distribution. Springer, New York, pp. 527-541. doi: 10.1007/978-1-4612-4380-9_37.

Cox, D. R., Oakes, D., 1984. Proportional hazard model. In: Analysis of survival data. CRC Press, pp. 91-110.

EFSA, 2015. European food safety authority and european centre for disease prevention and control. EFSA Journal. 13, 1-191. doi: $10.2903 /$ j.efsa.2015.4329

Gelman, A., Rubin, D. B., 1992. Inference from iterative simulation using multiple sequences. Statistical Science. 7, 457-511.

Gómez-Aldapa, C. A., del Refugio Torres-Vitela, M., Villarruel-López, A., Castro-Rosas, J., 2012. The role of foods in Salmonella infections. In: Salmonella-A Dangerous Foodborne Pathogen, pp. 21-46. doi:10.5772/28316

Hope, I. A., 1999. C. elegans: a practical approach. OUP Oxford. Volume 213. doi:10.1046/j.1365-2540.2000.0745d.x.

Ibrahim, J. G., Chen, M.-H., Sinha, D., 2001. Introduction. In: Bayesian Sur-

n vival Analysis. Springer-Verlag, New York, pp. 13-16.doi 10.1007/978-1-4757$3447-8$.

Kass, R. E., Carlin, B. P., Gelman, A., Neal, R., 1998. Markov Chain Monte Carlo in Practice: A Roundtable Discussion. The American Statistician. 52, 93-100.

Kisluk, G., Kalily, E., Yaron, S., 2013. Resistance to essential oils affects survival of Salmonella enterica serovars in growing and harvested basil. Environmental Microbiology. 15, 2787-2798. doi:10.1111/1462-2920.12139.

Klein, J. P., Moeschberger, M. L., 2003. Examples of survival data. In: Survival Analysis. Techniques for Censored and Truncated Data. Second Edition. Springer-Verlag, New York, pp. 1-19. 
Labrousse, A., Chauvet, S., Couillault, C., Kurz, C. L., Ewbank, J. J., 2000.

Caenorhabditis elegans is a model host for Salmonella typhimurium. Current Biology. 10, 1543-1545.

Leung, M. C., Williams, P. L., Benedetto, A., Au, C., Helmcke, K. J., Aschner, M., Meyer, J. N., 2008. Caenorhabditis elegans: an emerging model in biomedical and environmental toxicology. Toxicological sciences. 106, 5-28. doi:10.1093/toxsci/kfn121

Lunn, D. J., Thomas, A., Best, N., Spiegelhalter, D., 2000. WinBUGS - A Bayesian modelling framework: Concepts, structure, and extensibility. Statistics and Computing. 10, 325-337. doi:10.1023/A:1008929526011.

Majowicz, S. E., Musto, J., Scallan, E., Angulo, F. J., Kirk, M., O’Brien, S. J., Jones, T. F., Fazil, A., Hoekstra, R. M. et al., 2010. The global burden of nontyphoidal Salmonella gastroenteritis. Clinical Infectious Diseases. 50, 882-889. doi: $10.1086 / 650733$

Mosqueda-Melgar, J., Raybaudi-Massilia, R. M., Martín-Belloso, O., 2012. Microbiological shelf life and sensory evaluation of fruit juices treated by highintensity pulsed electric fields and antimicrobials. Food and Bioproducts Processing. 90, 205-214. doi:10.1016/j.fbp.2011.03.004.

Olaimat, A. N., Holley, R. A., 2012. Factors influencing the microbial safety of fresh produce: a review. Food Microbiology. 32, 1-19. doi:10.1016/j.fm.2012.04.016

O'Shea, N., Arendt, E. K., Gallagher, E., 2012. Dietary fibre and phytochemical characteristics of fruit and vegetable by-products and their recent applications as novel ingredients in food products. Innovative Food Science \& Emerging Technologies. 16, 1-10. doi $10.1016 /$ j.ifset.2012.06.002 M., 2007a. Inactivation of Enterobacter sakazakii by pulsed electric field in 
buffered peptone water and infant formula milk. International Dairy Journal. 17, 1441-1449. doi $10.1016 /$ j.idairyj.2007.04.007.

Pérez, P., Aliaga, R. D., Reyes, S. D., López, M. A., 2007b. Pressure inactivation kinetics of Enterobacter sakazakii in infant formula milk. Journal of Food Protection. $70,2281-2289$.

Rendueles, E., Omer, M., Alvseike, O., Alonso-Calleja, C., Capita, R., Prieto, M., 2011. Microbiological food safety assessment of high hydrostatic pressure processing: a review. LWT-Food Science and Technology. 44, 1251-1260.

Saldaña, G., Álvarez, I., Condón, S., Raso, J., 2014. Microbiological aspects related to the feasibility of pef technology for food pasteurization. Critical Reviews in Food Science and Nutrition. 54, 1415-1426.

Sanz-Puig, M., Moreno, P., Pina-Pérez, M. C., Rodrigo, D., Martínez, A., 2017. Combined effect of high hydrostatic pressure (HHP) and antimicrobial from ${ }_{420}$ agro-industrial by-products against $S$. typhimurium. LWT-Food Science and Technology. 77, 126-133. doi:10.1016/j.lwt.2016.11.031

Sanz-Puig, M., Pina-Pérez, M. C., Criado, M. N., Rodrigo, D., Martínez-López, A., 2015. Antimicrobial potential of cauliflower, broccoli, and okara byproducts against foodborne bacteria. Foodborne Pathogens and Disease. 12, 39-46. doi:10.1089/fpd.2014.180

Stiernagle, T., 2006. Maintenance of $C$. elegans. In: WormBook. The C. elegans Research Community, WormBook.

Van Boeijen, I. K., Casey, P. G., Hill, C., Moezelaar, R., Zwietering, M. H., Gahan, C. G., Abee, T., 2013. Virulence aspects of Listeria monocytogenes LO28 high pressure-resistant variants. Microbial Pathogenesis. 59, 48-51.

Vanlint, D., 2013. The evolution of bacterial resistance against high hydrostatic pressure. Ph.D. thesis. University of Reading (UK). 
Vieira, P. M., 2013. Avaliacão da composicão química, dos compostos bioativos e da atividade antioxidante em seis espécies de flores comestíveis. Ph.D. thesis. Universidade Estadual Paulista (UNESP).

Viuda-Martos, M., Ruiz-Navajas, Y., Fernández-López, J., Perez- Álvarez, J., 2008. Antibacterial activity of lemon (Citrus lemon 1.), mandarin (Citrus reticulata 1.), grapefruit (Citrus paradisi 1.) and orange (Citrus sinen-

¿ sis 1.) essential oils. Journal of Food Safety. 28, 567-576. doi:10.1111/j.1745440 4565.2008.00131.x. 